\title{
Amino Acids in Amniotic Fluid in the Second Trimester of Gestation
}

\author{
W. CHRISTINE MESAVAGE, SHARON F. SUCHY, DEBRA L. WEINER, CAROL S. NANCE, \\ DAVID B. FLANNERY, ${ }^{1}$ AND BARRY WOLF \\ Department of Human Genetics [W.C.M., S.F.S., D.L.W., C.S.N., D.B.F., B.W.] and Department of Pediatrics \\ [D.B.F., B.W.], Children's Medical Center, Medical College of Virginia, Richmond, Virginia 23298
}

\begin{abstract}
The concentrations of amino acids in amniotic fluid have been used in the prenatal diagnosis of several inherited metabolic disorders. However, previous studies have usually examined only a small number of control amniotic fluid samples. We have, therefore, measured the amino acids in amniotic fluid samples from 183 normal pregnancies between the 13th and 23 rd wk gestation of women ranging in age from 17 to $43 \mathrm{yr}$. The concentrations of Ala, Lys, Val, Glu, Pro, Thr, and Gly, in descending order, accounted for about $70 \%$ of the amino acids in amniotic fluids. A negative correlation with gestational age $(-0.34$ to -0.24$)$ was found for Leu, Val, Ile, Phe, Lys, Ala, Asp, Tyr, Glu, and Pro, with Leu showing the greatest rate of change. The concentration of GIn increased slightly $(r=0.18)$, whereas the other amino acids did not change significantly during this period. Statistically significant positive correlations, at all gestational ages, were observed among Val, Leu, and Ile. These branched-chain amino acids also correlated positively with Phe, Lys, Asp, Thr, Ser, Glu, Pro, Gly, Ala, and Tyr, and the amino acids within this group correlated with each other. Additionally, strong positive correlations were observed between Phe and Tyr and between Gly and Ser. No significant correlations were found between any of the amino acids and maternal age or fetal sex. Significant positive correlations between amino acids may be indicative of common transport or degradative pathways and suggest that maintenance of specific relative concentrations in amniotic fluid may be essential for normal fetal development. These data provide normative values for evaluating abnormal amino acid concentrations or ratios, which will be useful for studying their possible role as teratogenic agents as well as for the prenatal diagnosis of specific inborn errors of metabolism. (Pediatr Res 19: 1021-1024, 1985)
\end{abstract}

In the absence of a known or measurable enzyme defect, abnormal concentrations of free amino acids have been used in the prenatal diagnosis of some disorders of amino acid metabolism. For example, an elevated gly/ser ratio in amniotic fluid was used to diagnose prenatally nonketotic hyperglycinemia (1), the diagnosis of citrullinemia was made based on elevated concentrations of citrulline in the amniotic fluid of an affected fetus (2), and concentrations of argininosuccinic acid were elevated in

Received March 5, 1985; accepted May 23, 1985.

Correspondence Barry Wolf, M.D., Ph.D., Department of Human Genetics, Medical College of Virginia, P. O. Box 33, MCV Station, Richmond, VA 23298.

Paper 261 from the Department of Human Genetics of the Medical College of Virginia.

${ }^{1}$ Present address: Department of Pediatrics, Medical College of Georgia, Augusta, GA 30912. the amniotic fluid of a fetus with argininosuccinic aciduria (3). In addition, elevated concentrations of specific free amino acids have been observed in amniotic fluid of fetuses with Lowe's syndrome (4), central nervous system malformations $(5,6)$, and in fetuses whose mothers have diabetes mellitus (7). However, when a small number of control samples are analyzed, deviations from these control values must be interpreted cautiously. For example, Mesavage et al. (8), demonstrated that the gly/ser ratio of the affected fetus described by Garciá-Castro et al. (1) was within the normal range when a larger number of control samples was considered.

In order to better define abnormalities in the concentrations of amino acids in amniotic fluid and their potential value for prenatal diagnosis, we have analyzed the amino acid composition of amniotic fluids of 183 normal pregnancies. These data have also suggested interrelationships among various amino acids which may aid in understanding their role in fetal development.

\section{MATERIALS AND METHODS}

Amniotic fluid was collected from 183 normal pregnant women ranging in age from 17 to $43 \mathrm{yr}$ (mean $35.5 \mathrm{yr}$ ). Eightynine male and 93 female fetuses (the sex of one fetus was not reported) were studied at gestational ages between 13 and $23 \mathrm{wk}$ (mean $17.4 \mathrm{wk}$ ). Gestational ages were determined from biparietal diameter measurements obtained by ultrasonography $(2$ $\mathrm{SDs}=1.6-1.7 \mathrm{wk})$. Fluids obtained from women at risk for having a child with a metabolic disorder or whose pregnancy did not have a normal outcome were not included in the study.

Amniotic fluid samples were centrifuged at $140,000 \times g$ for $30 \mathrm{~min}$. The decanted supernatant was deproteinized with 20 $\mathrm{mg} / \mathrm{ml}$ of sulfosalicylic acid, recentrifuged, and the supernatant frozen at $-70^{\circ} \mathrm{C}$ until the time of analysis. Amino acid analysis was performed by column chromatography on a Joel 5-AH amino acid analyzer using a two-column lithium buffer system. After reaction with ninhydrin reagent, the amino acids in the column eluate were quantitated by their absorbance at 570 and $440 \mathrm{~nm}$ using an infotronics CRS-110A integrator. Amino acid standards were run daily with the amniotic fluid samples.

Mean concentrations and SDs were calculated for each amino acid at weekly gestational ages. Amino acids that eluted at the beginning or end of a run or at a buffer front were sometimes undetected; these were treated as missing values and were excluded from the analyses. Correlation coefficients were calculated between amino acids and between each amino acid and gestational age, maternal age, and the sex of the fetus. A $p$ value of less than 0.05 was considered significant.

Factor analysis with varimax rotation was performed on the amino acid data utilizing the SAS statistical package (9). To avoid deleting entire samples which contained missing values, the correlations were used in this analysis. 


\section{RESULTS}

The means, ranges, SDs, and percentages of total amino acid concentrations are presented for 23 free amino acids (Table 1). Ala and Lys constitute more than $30 \%$ of the total amino acid concentration, and Val, Glu, Pro, Thr, and Gly contribute an additional $40 \%$ of the total.

Correlations between amino acid concentrations are listed in Table 2. The highest positive correlations are between Val, Leu, and Ile. These branched-chain amino acids also correlate positively with Phe, Lys, Asp, Thr, Ser, Glu, Pro, Gly, Ala, Lys, and Tyr, and the amino acids within this group correlate with each other. In addition, strong positive correlations are observed between Phe and Tyr, and between Gly and Ser.

Leu, Ile, Val,. Phe, Lys, and Ala correlate negatively with gestational age $(-0.34$ to -0.24$)$ at the $p<0.01$ level of significance, whereas Tyr, Asp, Glu, and Pro correlate negatively at the $p<0.05$ level. The concentration of Leu has the highest correlation with gestational age $(r=-0.34)$. The concentration of Gln increases slightly with gestational age $(r=0.18)$. The remaining amino acids show no significant change with gestational age.

In order to further characterize the relative variation in amino acid concentrations, factor analysis was performed on the full sample. Three factors, having eigenvalues greater than unity in the initial run, were extracted. These three accounted for $41 \%$ of the variance. Table 3 shows the loading values on the first three factors, obtained by a varimax orthagonal rotation. Consideration of additional factors did account for more of the variance, but did not discriminate further among the groups.

No significant correlations are found between any of the amino acids and maternal age or fetal sex.

\section{DISCUSSION}

The amino acid concentrations were determined in 183 amniotic fluid samples obtained at 13 to $23 \mathrm{wk}$ gestation. The relative concentrations of the amino acids reported in other studies based on smaller sample sizes are consistent with our results $(10-12)$. The strong correlations observed between many of the neutral amino acids, particularly between the branched-

Table 1. Composition of amino acids in amniotic fluid samples of 183 normal pregnancies

\begin{tabular}{lccc}
\hline $\begin{array}{c}\text { Amino acid } \\
(n)\end{array}$ & $\begin{array}{c}\text { Mean } \pm 1 \mathrm{SD} \\
\text { concentration } \\
(\mu \mathrm{mol} / \mathrm{ml})\end{array}$ & $\begin{array}{c}\text { Range } \\
(\mu \mathrm{mol} / \mathrm{ml})\end{array}$ & $\begin{array}{c}\text { \% of total } \\
\text { amino acid } \\
\text { concentrations }\end{array}$ \\
\hline Ala (183) & $0.428 \pm 0.162$ & $0.050-1.54$ & 21.3 \\
Lys (180) & $0.282 \pm 0.108$ & $0.010-0.70$ & 10.5 \\
Val (182) & $0.182 \pm 0.087$ & $0.000-0.65$ & 9.4 \\
Glu (180) & $0.225 \pm 0.143$ & $0.000-0.99$ & 7.5 \\
Pro (176) & $0.205 \pm 0.098$ & $0.050-1.13$ & 7.5 \\
Thr (183) & $0.170 \pm 0.096$ & $0.000-0.73$ & 6.7 \\
Gly (183) & $0.168 \pm 0.080$ & $0.010-0.91$ & 4.9 \\
Asn (168) & $0.229 \pm 0.239$ & $0.010-1.47$ & 4.5 \\
Tau (176) & $0.105 \pm 0.116$ & $0.012-0.98$ & 4.1 \\
Gln (169) & $0.201 \pm 0.211$ & $0.000-1.34$ & 3.7 \\
Leu (182) & $0.082 \pm 0.044$ & $0.002-0.32$ & 3.7 \\
His (181) & $0.128 \pm 0.100$ & $0.020-0.97$ & 2.6 \\
Phe (167) & $0.060 \pm 0.026$ & $0.006-0.21$ & 2.5 \\
Tyr (181) & $0.058 \pm 0.054$ & $0.017-0.07$ & 2.3 \\
Cys (178) & $0.065 \pm 0.072$ & $0.001-0.58$ & 2.0 \\
lle (181) & $0.037 \pm 0.020$ & $0.001-0.16$ & 1.9 \\
Arg (146) & $0.069 \pm 0.160$ & $0.003-1.91$ & 1.8 \\
Met (181) & $0.035 \pm 0.059$ & $0.001-0.45$ & 1.0 \\
Orn (157) & $0.039 \pm 0.039$ & $0.005-0.37$ & 1.0 \\
Ser (183) & $0.020 \pm 0.019$ & $0.001-0.17$ & 0.6 \\
Asp (180) & $0.004 \pm 0.003$ & $0.001-0.04$ & 0.2 \\
Cysh (171) & $0.008 \pm 0.009$ & $0.001-0.06$ & 0.1 \\
Trp (112) & $0.042 \pm 0.084$ & $0.001-0.66$ & 0.1 \\
\hline & & &
\end{tabular}

chain amino acids, indicate that this group may be regulated by common mechanisms, such as placental or renal tubular transport systems or metabolic pathways, or may be modulated by factors which affect specific transport systems or metabolic pathways. Factor analysis of these data support this hypothesis. There appears to be one factor that contributes to the variation in the concentrations of Lys, Ala, Val, Ile, Leu, and Phe, all of which share a common transport system (13). In addition, Val, Ile, and Leu undergo degradation by the same enzyme complex. A second factor contributes to variations in Ser, Gly, and Phe concentrations, three amino acids which also share a transport system (13). Moreover, Gly and Ser are interconverted by a single enzyme. Variations in Arg and Cysh concentrations are attributable to a third factor.

There appear to be at least five amino acid transport systems in the mammalian kidney (13); two for the transport of neutral amino acids, one for the transport of basic amino acids, one for the transport of amino acids and Gly, and one for acidic amino acid transport. However, all of the amino acids show some overlap in specificity among the transport systems. For example, the overlap between the two transport systems for the neutral amino acids is so extensive that nearly all of the neutral amino acids are transported, with differing affinities, by both systems. This may partially explain the lack of correlation between some of them. In addition to these high capacity, low specificity systems, there may also be low capacity, high specificity systems which would transport only one or two amino acids. Therefore, not only do some amino acids interact with more than one main transport system, but more specialized carriers may exist for individual amino acids within each group. The strong correlations found between Gly and Ser and between Phe and Tyr indicate that common metabolic pathways may also influence amino acid concentrations.

Our observation of decreases in the concentrations of amino acids with gestational age is in agreement with most other reports $(5,12,14,15)$. The amino acid concentrations in our study decreased by 24 to $61 \%$ between 13 and 20 wk gestation, whereas in the study by Scott et al. (12), these decreases ranged from 48$85 \%$ over the same gestational period (gestational ages were determined by crown-rump measurements). Scott also reported higher correlations between gestational age ( 7 to $20 \mathrm{wk}$ ) and the concentrations of Val, Phe, Ile, Leu, Tyr, Ala, Met, Ser, Orn, Arg, Lys, Pro, Tau, and His $(r=-0.66$ to -0.89$)$. Only a few studies have reported increases in amino acid concentrations with gestational age, specifically in the concentrations of serine (16), ethanolamine and taurine (14), and cysteic acid $(5,17)$. We observed a significant increase only in Gln concentration.

Decreases in amino acid concentrations in amniotic fluid with gestational age may reflect physiologic changes which result from or affect normal developmental processes. As pregnancy progresses, there are decreases in the concentrations of amino acids in fetal urine compared to the concentrations in fetal blood (10). This may be due principally to greater renal tubular selectivity which occurs by the 10th to 12 th wk of gestation.

Most of the amino acids in amniotic fluid are of fetal origin $(18,19)$. There may be some direct transfer of amino acids across the chorioamnion, placental, and umbilical surfaces, fetal skin, salivary glands, lung fluid, intestinal secretions, and Wharton's jelly, lying between the chorion and amnion (18). Other factors which are related to the nutritional state of the mother, including the concentrations of potassium, glucose, sulfate, and phosphate, may influence the transport of amino acids and alter the amino acid concentration in amniotic fluid (13). These factors may partly explain the wide variations found in amino acid concentrations in amniotic fluid of fetuses of the same gestational age and emphasize the need for a sufficient sample size to take into account normal variations. Thus, it is probable that multiple factors regulate amino acid concentrations in amniotic fluid.

Amino acid homeostatis in amniotic fluid may be essential for normal fetal growth and development. Abnormal amino acid 


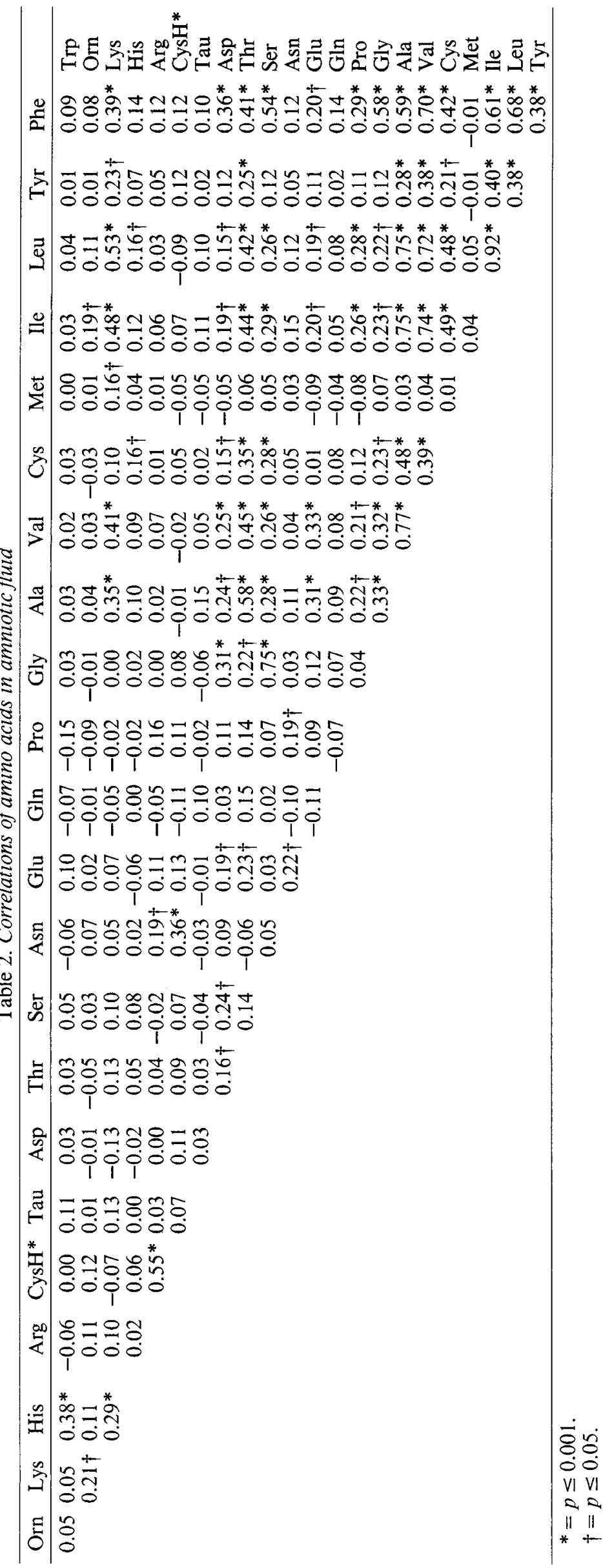


Table 3. Factor analysis of amino acids in amniotic fluid

\begin{tabular}{ccc}
\hline Factor & Amino acid & Loading value* \\
\hline I & Leu & 0.93 \\
& Ile & 0.88 \\
& Ala & 0.77 \\
Val & 0.77 \\
& Phe & 0.58 \\
& Lys & 0.55 \\
II & Gly & 0.92 \\
& Ser & 0.73 \\
& Phe & 0.63 \\
III & Cysh & 0.88 \\
& Arg & 0.59 \\
\hline
\end{tabular}

* Only amino acids with a loading value of greater than 0.5 are included.

concentrations or amino acid ratios may indicate abnormal fetal development which may be caused by genetic defects, such as maternal phenylketonuria, or exposure to teratogenic agents. Although the relative concentrations of amino acids are consistent among laboratories, the actual concentrations frequently differ. Therefore, if prenatal diagnosis is to be attempted it is essential that each laboratory determine its own normal concentration ranges or relative concentration ranges using a large number of control samples.

Acknowledgements. The authors thank Dr. W. E. Nance, Dr. Lindon J. Eaves, and H. Metzler for thoughtful discussions, and Rosa M. Vaughan for her excellent secretarial assistance.

\section{REFERENCES}

1. Garciá-Castro JM, Isales-Forsythe CM, Levy HL, Shih VE, Laó-Vélez CR, Gónzalez-Riós M, Torres LC 1982 Prenatal diagnosis of nonketotic hyperglycinemia. N Engl J Med 306:79-81

2. Fleisher LD, Harris CJ, Mitchell DA, Nadler HL 1983 Citrullinemia: Prenatal diagnosis of an affected fetus. Am $\mathbf{J}$ Hum Genet 35:85-90

3. Goodman SI, Mace JW, Turner B, Garrett WJ 1973 Antenatal diagnosis of arginino-succinic aciduria. Clin Genet 4:236-242

4. Tada K, Higami S, Fujimoto A, Ogita Y 1974 Free amino acid levels in amniotic fluid of fetuses affected with Lowe's syndrome or phenylketonuria. Tohoku J Exp Med 113:169-171

5. Emery AEH, Burt D, Scrimgeour JB 1973 Amino acid composition of amniotic fluid in central-nervous-system malformations. Lancet 1:970-971

6. Kumar S, Foreman JR, Rathi M 1980 Amniotic fluid and maternal serum amino acid levels in malformations of fetal central nervous system. Neurochem Res 5:453-458

7. Horská S, Ráová M, Vondrácek J 1980 Amino acids in the amniotic fluid of diabetic mothers. Biol Neonate 37:204-208

8. Mesavage C, Nance CS, Flannery DB, Weiner DL, Suchy SF, Wolf B 1983 Glycine/serine ratios in amniotic fluid: an unreliable indicator for the prenatal diagnosis of nonketotic hyperglycinemia. Clin Genet 23:354-358

9. Helwig JT, Council KA (eds) 1979 SAS User's Guide. SAS Institute Inc, Cary, $\mathrm{NC}$, pp 203-210

10. A'Zary E, Saifer A, Schneck L 1973 The free amino acids in maternal and fetal extracellular fluids collected during early pregnancy. Am $\mathbf{J}$ Obstet Gynecol 116:854-866

11. Saifer A, A'Zary E, Valenti C, Schneck L 1970 Quantitative cation-exchange chromatographic analysis of free amino acids in human amniotic fluid collected during early pregnancy. Clin Chem 16:891-895

12. Scott CR, Teng CC, Sagerson RN, Nelson T 1973 Amino acids in amniotic fluid: changes in concentration during the first half of pregnancy. Pediatr Res 6:659-663

13. Young JA, Freedman BS 1971 Renal tubular transport of amino acids. Clin Chem 17:245-262

14. O'Neill RT, Morrow G, Hammel D, Auerbach VH, Barness LA 1971 Diagnostic significance of amniotic fluid amino acids. Obstet Gynecol 37:550554

15. Thomas GH, Parmley TH, Stevenson RE, Howell RR 1971 Developmental changes in amino acid concentrations in amniotic fluid: abnormal findings in maternal phenylketonuria. Am J Obstet Gynecol 111:38-42

16. Schulman JD, Queenan JT, Doores L 1972 Gas chromatographic analysis of concentrations of amino acids in amniotic fluid from early, middle, and late periods of human gestation. Am J Obstet Gynecol 114:243-249

17. Kang ES, Scalon J 1974 Concentrations of the free amino acids in human amniotic fluid during normal and abnormal pregnancies. Am $\mathbf{J}$ Obstet Gynecol 119:603-609

18. Cockburn F, Robins SP, Forfar JO 1970 Free amino acid concentrations in fetal fluids. Br Med J 3:747-750

19. Reid DW, Campbell DJ, Yakymyshyn LY 1971 Quantitative amino acids in amniotic fluid and maternal plasma in early and late pregnancy. Am J Obstet Gynecol 111:251-258 